\title{
Single and Multipinhole Collimator Design Evaluation Method for Small Animal SPECT
}

\author{
Kathleen Vunckx ${ }^{1}$, Dirk Bequé ${ }^{1}$, Michel Defrise ${ }^{2}$ and Johan Nuyts ${ }^{1}$
}

\begin{abstract}
High resolution functional imaging of small animals is often obtained by single pinhole collimated SPECT with a circular acquisition orbit. However, the acquired data are incomplete. Multipinhole SPECT has the advantage of adding more information due to its improved sampling and possibly increases the resolution and the sensitivity. To evaluate different pinhole collimator designs a method is needed that tells how much information is missing, or equivalently, how well the animal can be reconstructed. In this paper we propose two methods to facilitate the evaluation process for single and multipinhole collimator designs. Firstly, we examine the quality of each postprocessed MLEM reconstructed voxel by studying its linearized local impulse response (LLIR) and its (co)variance for a predefined target resolution. Secondly, since increasing the number of pinhole apertures may cause the projections to overlap, point and circular artifacts can occur in the reconstruction image. The position of these 'ghost points' and 'ghost circles' can be well predicted analytically. We also give some rules of thumb to estimate the (relative) intensity of the artifacts. The methods are validated with simulations.
\end{abstract}

\section{INTRODUCTION}

Single pinhole collimated SPECT is regularly used nowadays for high quality functional imaging of small animals. Despite the high resolution, the sensitivity is rather low and the data acquired with a circular orbit have been proven to be incomplete [1]. The use of a multipinhole collimator can increase the sensitivity without loss of resolution and the additional information can reduce the incompleteness. Mathematical analysis can only verify whether the data are complete or not [1]. To be able to compare different single and multipinhole collimator designs a method is needed that quantifies how much information is missing. Pinhole collimator designs are usually compared based on their resolution and their sensitivity, on Monte Carlo simulations or on image reconstructions [2]-[4].

In this paper we propose a new evaluation technique for single and multipinhole collimator designs. In our method collimator designs are evaluated with respect to the quality of each reconstructed voxel, and the occurrence of reconstruction artifacts due to overlapping projections.

1 K. Vunckx and J. Nuyts are with the Dept. of Nuclear Medicine, K.U.Leuven, B-3000 Leuven, Belgium. D. Bequé was with the Dept. of Nuclear Medicine, K.U.Leuven, B-3000 Leuven, Belgium. He is now with G.E. Global Research Europe, D-85748 Garching, Germany.

${ }^{2}$ M. Defrise is with the Dept. of Nuclear Medicine, V.U.Brussel, B-1090 Brussel, Belgium.

This work is supported by F.W.O. grant G.0174.03, K.U.Leuven grant IDO/02/012, I.W.T. grant SBO - ANIMONE and NIH GRANT R13 EB005531.

e-mail: kathleen.vunckx@uz.kuleuven.be
Quantifying the quality of each reconstructed voxel is typically done by exploring the properties of the linearized local impulse response (LLIR) of each voxel. In order to achieve a fixed (and more isotropical) target resolution for the LLIRs, we replace the usual MAP approximations [5]-[8] by efficient post-processed MLEM approximations.

In practical multipinhole collimator design, overlapping projections frequently result in artifacts (both points and circles) in the reconstruction image. To be able to predict the occurrence of these artifacts for a particular collimator design, we study the origin of these artifacts and develop a method to predict their position in the reconstruction image.

Based on the voxel quality quantification (section II-A) and the prediction of ghost points and ghost circles (section IIB), we propose a method in section II-C to find a satisfying multipinhole collimator design for a particular application and a fixed target resolution. In section III some experiments are described to validate the voxel quality quantification method and the artifact prediction method. The results of these experiments are presented in section IV and are further discussed in section V. Section VI briefly summarizes the presented work.

\section{THEORY}

\section{A. Reconstruction Quality Quantification}

The regular impulse response theory is not applicable for the maximum likelihood (ML) or maximum a posteriori (MAP) reconstruction of emission tomography, since the reconstruction $\hat{\Lambda}$ of the unknown activity distribution $\Lambda$ is space-variant, object-dependent and nonlinear in the projection data $Q$. Fessler et al. [5] proposed a generalization of the impulse response for emission tomography, called the linearized local impulse response (LLIR) $l^{j}$ :

$$
\begin{aligned}
l^{j}(\hat{\Lambda}) & =\lim _{\delta \rightarrow 0} \frac{\hat{\Lambda}\left(\bar{Q}\left(\Lambda+\delta e^{j}\right)\right)-\hat{\Lambda}(\bar{Q}(\Lambda))}{\delta} \\
& =\frac{\delta}{\delta \lambda_{j}} \hat{\Lambda}(\bar{Q}(\Lambda))
\end{aligned}
$$

where $\bar{Q}$ is the expectation of $Q$ and $e^{j}$ is the $j$-th unit vector. Fessler et al. [5], [9] formulated the following approximations to calculate the LLIR and its covariance matrix after convergence of a MAP reconstruction:

$$
\begin{aligned}
l^{j}(\hat{\Lambda}) & \approx[\boldsymbol{F}+\beta \boldsymbol{U}]^{-1} \boldsymbol{F} e^{j} \\
\operatorname{Cov}^{j}(\hat{\Lambda}) & \approx[\boldsymbol{F}+\beta \boldsymbol{U}]^{-1} \boldsymbol{F}[\boldsymbol{F}+\beta \boldsymbol{U}]^{-1} e^{j}
\end{aligned}
$$

where $\boldsymbol{F}$ is the Fisher information matrix, which is a function of $\bar{Q}, \beta$ is the smoothing parameter and $\boldsymbol{U}$ is the quadratic prior, used for regularization. 
To compare different pinhole designs we want to use an iterative reconstruction method with a fixed target resolution. For MAP reconstruction Stayman et al. [10] proposed a method that yields reconstructed images with isotropic, uniform resolution. To validate our approximations, we want to impose isotropic uniform resolution in an easy way. Therefore we replaced the MAP reconstruction by post-processed MLEM. The post-processing filter $P$ can be found from $T \approx P G F$, with $T$ a Gaussian with a full width at half maximum (FWHM) equal to the target resolution and $G$ a pseudoinverse of $F$. The LLIR and its covariance then become:

$$
\begin{aligned}
l^{j}(\hat{\Lambda}) & \approx \boldsymbol{P} \boldsymbol{G F} e^{j} \\
\operatorname{Cov}^{j}(\hat{\Lambda}) & \approx \boldsymbol{P} \boldsymbol{G F G P} \boldsymbol{P}^{\boldsymbol{T}} e^{j}
\end{aligned}
$$

where ${ }^{T}$ denotes transpose.

The calculation of the LLIR in equation (5) requires the inversion of the $J_{\mathrm{X}} J$ matrix $F$, in which $J$ represents the total number of image voxels. Given the relatively high number of image voxels in emission tomography, this solution is not feasible in practice. If $F$ would be shift-invariant, then equation (5) would reduce to the subsequent convolution of the perturbation $e^{j}$ with the convolution masks defined by $F, G$ and $P$. However, single and multipinhole SPECT projections are not shift-invariant, so neither is $F$. One could however assume that the Fisher information matrix $F$ is locally shift-invariant. Therefore only a small error is made near the impulse when the above mentioned convolutions are performed. The calculation of the inverse can be done even more efficiently in the Fourier domain. In this way we get new approximations, that are much more efficient than equations (5) and (6). The contrast recovery coefficient (CRC) and the variance of the LLIR are given by the $j$-th element of $l^{j}$ and $\operatorname{Cov}^{j}$, respectively:

$$
\begin{aligned}
l_{j}^{j}(\hat{\Lambda}) & \approx e^{j T} \boldsymbol{P} \tilde{\boldsymbol{G}} \tilde{\boldsymbol{F}} e^{j} \\
\operatorname{Cov}_{j}^{j}(\hat{\Lambda}) & \approx e^{j T} \boldsymbol{P} \tilde{\boldsymbol{G}} \tilde{\boldsymbol{F}} \tilde{\boldsymbol{G}} \boldsymbol{P}^{T} e^{j}
\end{aligned}
$$

where $\tilde{F}$ and $\tilde{G}$ are the local shift-invariant based approximations of $F$ and $G$, respectively. This local shift-invariant assumption has been proposed earlier in [7], [11] in the context of shift-variant PET, and in [8] for multipinhole.

The CRC can be seen as a measure of resolution [6]. Since we work with a fixed target resolution, we only have to verify that the CRC stays at a more or less constant value. The variance of the LLIR can then be used as a quality measure of the reconstruction of that voxel. An alternative measure is the contrast-to-noise ratio (CNR), which is defined by:

$$
C N R=\frac{C R C}{\sqrt{\text { variance }}}
$$

\section{B. Prediction of 'Ghost Points' and 'Ghost Circles'}

When using a collimator with multiple pinholes the sensitivity of these pinhole apertures may overlap. This means that projected activity in this overlapping area can be explained by more than one activity distribution, which results in artifacts.

If two pinhole apertures lie on a line parallel to the axis of rotation, a point source (in a background) can cause

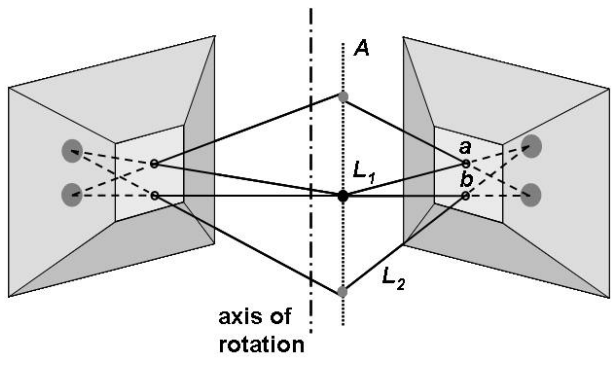

(a) Ghost points.

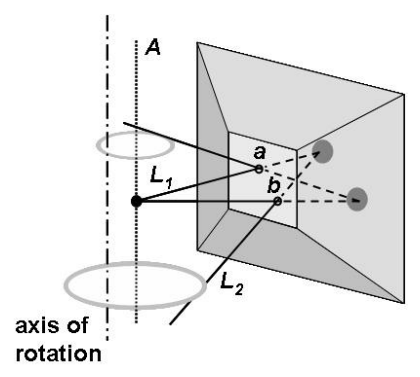

(b) Ghost circles.

Fig. 1. Ghost points and ghost circles.

two additional artificial activity points ('ghost' points) in the reconstructed image, if these points lie in the field of view (FOV) (see figure 1(a)). All other pairs of pinhole apertures cause two additional artificial activity circles ('ghost' circles), again if these lie in the FOV (see figure 1(b)). The position of these artifacts can be easily predicted. For each ordered pair of pinhole apertures $(a, b)$ one can calculate, for each projection angle, a point that belongs to a ghost point or a ghost circle. This can be done as follows:

- Calculate the projection of the point source through aperture $a$ on the detector (see $L_{1}$ in figure 1).

- Calculate the direction coefficient of the backprojection line ( $L_{2}$ in figure 1) through aperture $b$.

- Calculate the point on $L_{2}$ closest to line $A$ (through the point source, parallel to the axis of rotation)

All gathered points for one ordered pair of pinhole apertures form a ghost point or ghost circle ${ }^{1}$. In fact, a ghost circle is the enveloping curve of all lines L2.

At this time we have no algorithm to accurately predict the intensity. However, we can give some rules of thumb to estimate the (relative) importance of the artifacts. When the radius of a ghost circle increases, its intensity decreases. The intensity is also a function of the direction of the backprojection line $L_{2}$. The smaller the angle this line makes with the plane of the ghost circle, the higher its contribution to it and thus the higher the intensity of the circle. The intensity of a ghost point is of the same order as the intensity of the real point source

\footnotetext{
${ }^{1}$ These ghost 'points' are rather a collection of points which form a small blob. The ghost 'circles' are not always real circles, but can also be more skewed ellipses for example.
} 
(but always lower) and can therefore never be tolerated. In conclusion, if overlap occurs, the positioning of the pinholes should be done in a way that (a) no ghost points can be caused in the FOV (because these have a relatively high intensity) and (b) ghost circles that can occur in the FOV have a large radius. In addition these artifacts have a cumulative effect. Parallel positioning of aperture pairs should thus be avoided as well. Irregular pinhole patterns seem therefore better from an artifact point of view.

\section{Collimator Optimization Method}

To find a good pinhole collimator design for a given application, we propose the following recipe:

- Build a large set of collimator designs which differ in focal length, number of pinholes, pinhole aperture diameter(s), pinhole acceptance angle(s), pinhole inclination angle(s) and positioning of the pinhole(s).

- Check the reconstruction image quality of all (or a subset of) voxels of a software phantom (e.g. of (the head of) a rat) by calculating the variance or the contrast-to-noise ratio of their linearized local impulse response.

- If necessary, adapt the positioning of the pinholes slightly so that no ghost points nor small ghost circles appear in the field of view.

\section{EXPERIMENTS}

\section{A. Comparison of Different Single Pinhole Collimator De- signs}

To illustrate the use of the reconstruction quality quantification method described in section II-A we apply it to 8 different single pinhole collimator designs. The aperture diameter ranges from $0.6 \mathrm{~mm}$ to $2.0 \mathrm{~mm}$, the acceptance angle is $120^{\circ}$ and the focal distance (the distance between the detector and the aperture) is $85 \mathrm{~mm}$. The tungsten collimator insert is $6 \mathrm{~mm}$ thick. The sensitivity is modeled analytically using a ray tracing method, but without pinhole penetration. No scatter nor attenuation model is used.

The simulated SPECT scanner has one detector $(52 \times 52$ pixels of $2.0 \mathrm{~mm}$ in both directions) with an intrinsic resolution of $4.0 \mathrm{~mm}$. The distance ${ }^{2}$ between the detector and the axis of rotation (AOR) is $105.5 \mathrm{~mm}$. A perfect circular orbit with 64 equally spaced projection angles (60 sec each) is assumed.

The reconstructed image space contains 40x40x40 voxels of $0.6 \mathrm{~mm}$ is all directions. The phantom used for this simulation is a homogeneous sphere with a radius of $9.3 \mathrm{~mm}$. To be realistic each voxel is filled with an activity of $7.5 \mathrm{kBq}$. An impulse of $7.5 \mathrm{kBq}$ is given to an excentrical point at $(0.3$, $3.9,3.9$ ) in $\mathrm{mm}$.

The simulated data are reconstructed using post-processed MLEM with relaxed ordered subsets (OS) [12], [13]. An equivalent of 77 iterations are executed. Resolution recovery is done by the 7-ray method developed by Andreyev et al. [14]. The target resolution is set to $2.0 \mathrm{~mm}$.

\footnotetext{
${ }^{2}$ Most geometric parameters of the simulated SPECT scanner are rescaled to reduce the calculation time.
}

To validate our approximate method we calculate the CRC, the variance and the CNR, using equations (7)-(9). We compare these with the same measures found from the LLIR calculated from post-processed MLEM reconstructions. To get the LLIR we need to reconstruct the software phantom with and without an extra impulse in voxel $j$ :

$$
l^{j}(\hat{\Lambda})=\frac{\text { recon. with impulse }- \text { recon. without impulse }}{\text { impulse }}
$$

The variance is calculated based on 300 noise realizations.

\section{B. Position Prediction of Ghost Points and Ghost Circles}

To prove that the method for the prediction of the position of ghost points and ghost circles, explained in section II-B, is very accurate, a point source reconstruction is simulated for both kinds of artifacts and compared with the prediction.

Two pinholes on the AOR are known to cause two ghost points (figure 1(a)). To get both in the FOV, we put the apertures at a distance of $15 \mathrm{~mm}$ from each other. One aperture is positioned centrally in front of the detector. To produce two ghost circles in the FOV, we rotate the second aperture over $60^{\circ}$ around the first one. The pinholes are all focussed at a point at $50 \mathrm{~mm}$ from the central one (i.e. at a point on the AOR). For all apertures the diameter is $1.5 \mathrm{~mm}$, the acceptance angle is $60^{\circ}$ and the focal distance is $170 \mathrm{~mm}$. The tungsten collimator insert is $6 \mathrm{~mm}$ thick. The sensitivity is modeled including pinhole penetration. No scatter nor attenuation model is used.

We simulated a SPECT scanner with one detector $(256 \times 196$ pixels of $2 \mathrm{~mm}$ in both directions). The distance between the detector and the AOR is $220 \mathrm{~mm}$. Again a perfect circular orbit with 64 equally spaced projection angles is assumed.

A homogeneous sphere with $25 \mathrm{~mm}$ radius is reconstructed in an image space with $60 \times 60 \times 60$ voxels of $1 \mathrm{~mm}$ in each direction. The activity in the point source (at $(0.5,0.5,-5.5)$ in $\mathrm{mm}$ ) is twice the activity in each voxel of the sphere.

The same reconstruction algorithm is used as in section IIIA, but without post-processing. Since MLEM is very good at reconstructing point sources, we included the homogeneous spherical background, specified above. Then the reconstruction was performed as described in equation (10).

\section{RESUlTS}

\section{A. Comparison of Different Single Pinhole Collimator De- signs}

For the single pinhole SPECT setups (with an aperture diameter between $0.6 \mathrm{~mm}$ and $2.0 \mathrm{~mm}$ ), as described in section III-A, the results are displayed in figure 2 . The results acquired by executing the post-processed MLEM reconstructions are displayed as red diamonds. The black stars represent the results obtained from the approximate but much more efficient method. There is a very good agreement between these two methods, which indicates that the approximate method can be used in the future for the evaluation of other designs.

Figure 2(a) shows the contrast recovery coefficient of the LLIR for the 8 collimator designs. When the target resolution 


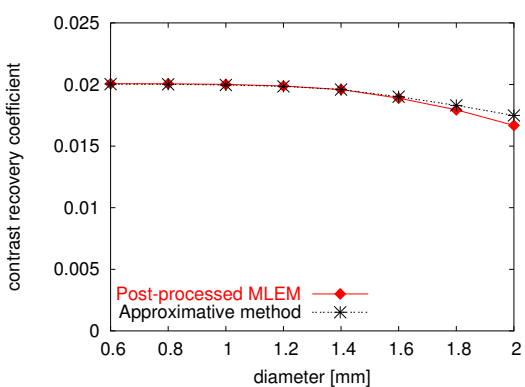

(a) Contrast recovery coefficient.

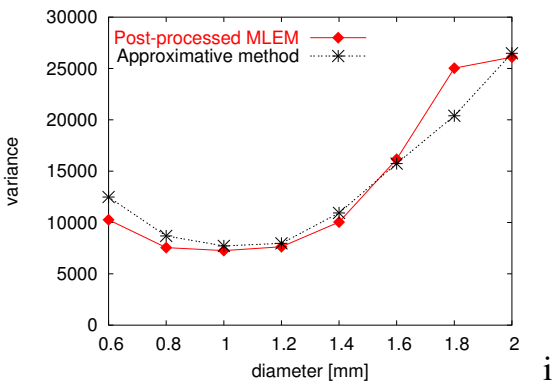

(b) Variance.

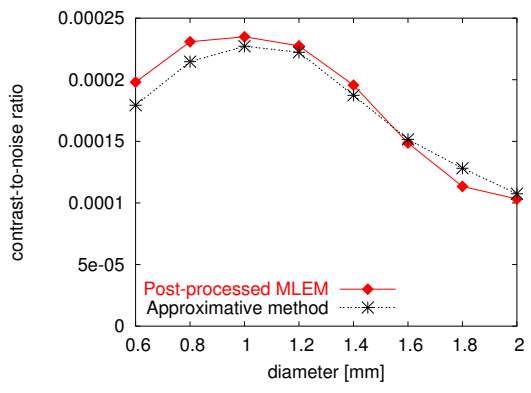

(c) Contrast-to-noise ratio.

Fig. 2. Illustration and validation of the reconstruction quality quantification method. Comparison of different single pinhole collimator designs.

cannot be reached anymore, the CRC starts to decrease. So from this curve we can conclude that pinholes with an aperture diameter larger than $1.4 \mathrm{~mm}$ do not satisfy the requirements. These can therefore be ignored in the next step.

The variance of the LLIR is shown in figure 2(b). The variance increases with increasing aperture diameter, due to increased sensitivity, and with increased post-smoothing. Since we require a fixed target resolution, the increasing diameter has to be compensated by reduced post-smoothing or even by deconvolution. This results in a minimum variance at a certain aperture diameter, and an increasing variance for larger diameters. The optimum for the examined designs is then found for a single pinhole with an aperture diameter of $1.0 \mathrm{~mm}$.

As an alternative measure the CNR could be used (equa- tion (9)). As can be seen in figure 2(c) the same optimum is found.

In conclusion we can say that there is a very good agreement between the analytical prediction and the simulations.

\section{B. Position Prediction of Ghost Points and Ghost Circles}

In this section we compare the ghost points and circles found by simulation with those of the prediction. The specifications of the pinhole SPECT systems and the point sources where described in section III-B. The results are displayed in figure 3 and 4, respectively. An excellent agreement is seen in all cases.

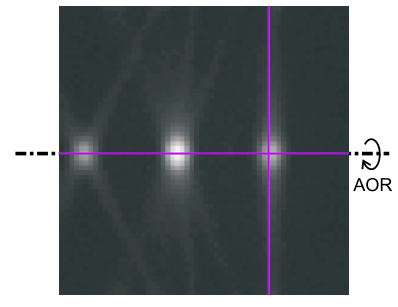

(a) Simulation, sagittal.

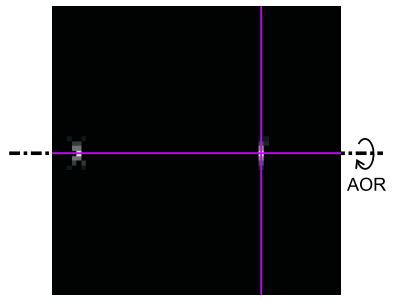

(b) Prediction, sagittal.
Fig. 3. Validation of the prediction of the position of two ghost points.

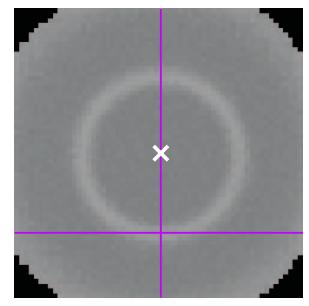

(a) Simulation, transaxial

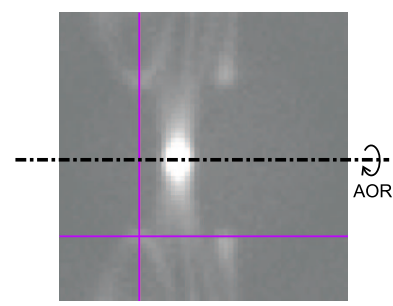

(c) Simulation, sagittal.

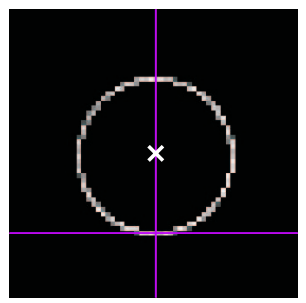

(b) Prediction, transaxial.

(d) Prediction, sagittal.

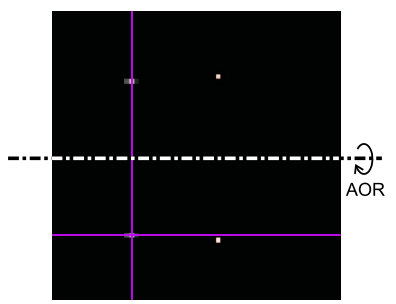

Fig. 4. Validation of the prediction of the position of two ghost circles.

\section{Discussion}

Although it has not been thoroughly tested yet, the reconstruction quality quantification method, presented in section IIA, should also be valid for (multi head) multi pinhole SPECT. This method can be applied to PET and other SPECT applications as well. 
The main difference with similar methods [6]-[8] seems to be the use of post-processed MLEM instead of MAP. However, if the regularization term $\beta U$ is designed in such a way that $[F+\beta U]^{-1}=P G$, like in [10], the difference between MAP and post-processed MLEM fades away and equations (3) and (4) become equations (5) and (6). We prefered to use post-processed MLEM, because we wished to obtain predefined isotropic resolution in an easy way to validate the approximations. Due to the uniform resolution, we only have to optimize one parameter, i.e. the variance of the LLIR in the voxel.

Some previously published methods, like [6], go even further in their approximations. They try to isolate shift-invariant terms, like the geometric projection matrix. Since it is the same for all voxels, it only has to be calculated once. This saves a lot of computation time. For pinhole system, this is of no use, because this matrix is shift-variant in that case. Another possibility is to isolate the matrices that are independent of the object, like the camera geometry matrix in [7]. This might be possible for a pinhole system, but it is of no use for us, since we test each camera geometry (including pinhole design) only once. Therefore, we have to use our previously stated approximations that are a less efficient, but still much faster than inverting the system matrix in the spacial domain.

If the software phantom is e.g. a homogeneous sphere, it is sufficient to investigate all voxels of a half plane. Because of the circular symmetry of the reconstruction properties this half plane provides all information about the reconstruction of the entire sphere. For a central single pinhole or a multipinhole design that is symmetrical with respect to the central transaxial plane, a quarter plane is even enough. Furthermore, the less regular the software phantom, the more voxels should be examined.

To get a fixed resolution over the object, the post-processing filter has to be calculated for each voxel seperately, since pinhole SPECT is space-variant. This is just to find the optimal design. It might not be practical to do this for reconstructing real data.

The optimum aperture diameter found in section IV-A is far below the target resolution. This is in agreement with [15]. The optimum is rather flat. It may be better to take a slightly larger aperture diameter to reduce scatter.

Instability problems still occur when testing diameters that are large with respect to the target resolution, but these are not relevant anyway, since the target resolution cannot be reached for these diameters.

To model the intensity distribution in the ghost points and circles, we spread the intensity over the backprojection line by Gaussian smoothing. Some minor correlation has been found between the predicted intensity and that found by reconstructing point sources. It has not been investigated more thoroughly, however. The effects of the point source-tobackground activity ratio and of the number of iterations have not been examined either.

Our current multipinhole design consists of 7 apertures in a very regular composition (a regular hexagonal) with 3 apertures on a line parallel to the axis of rotation. This seems a bad idea in the context of ghost artifacts. Next time we will probably choose for a more irregular pinhole collimator design and avoid aperture pairs on a line parallel to the axis of rotation.

\section{CONCLUSION}

An accurate and efficient method is proposed to evaluate different single and multipinhole collimator designs for a particular application and a fixed target resolution. It consists of two complementary methods. Firstly, the quality of the reconstruction of each voxel can be quantified efficiently based on the contrast recovery coefficient and the variance of its linearized local impulse response. Secondly, a new kind of image artifacts is described and a method to predict their position has been presented. Some basic rules about their relative intensity are given as well. Both methods have been validated using MLEM reconstructions. In the near future a multipinhole collimator will be designed to image rat brains with a dual head SPECT system.

\section{REFERENCES}

[1] H. K. Tuy, "An inversion formula for cone-beam reconstruction." SIAM J. Appl. Math., vol. 43(3), pp. 546-552, 1983.

[2] M. Ivanovic, D. A. Weber, and S. Loncaric, "Multi-pinhole collimator optimization for high resolution SPECT imaging." IEEE Med. Imag. Conf. Rec. 1997, vol. 2, pp. 1097-1101, 1997.

[3] N. U. Schramm, G. Ebel, U. Engeland, T. Schurrat, M. Béhé, and T. M. Behr, "High-resolution SPECT using multipinhole collimation." IEEE Trans. Nucl. Sci., vol. 50(3), pp. 315-320, 2003.

[4] M. F. Smith, S. R. Meikle, S. Majewski, and A. G. Weisenberger, "Design of multipinhole collimators for small animal SPECT." IEEE Nucl. Sci. Symp. and Med. Imag. Conf. 2003, Portland, OR, 2003.

[5] J. A. Fessler and W. L. Rogers, "Spatial resolution properties of penalized-likelihood image reconstruction: space-invariant tomographs." IEEE Trans. Image Proc., vol. 5(9), pp. 1346-1358, 1996.

[6] J. Qi and R. M. Leahy, "A theoretical study of the contrast recovery and variance of MAP reconstructions from PET data." IEEE Trans. Med. Imag., vol. 18(4), pp. 293-305, 1999.

[7] J. Qi and R. M. Leahy, "Resolution and noise properties of MAP reconstruction for fully 3-D PET." IEEE Trans. Med. Imag., vol. 19(5), pp. 493-506, 2000.

[8] L. J. Meng, W. L. Rogers, N. H. Clinthorne, and J. A. Fessler, "Feasibility study of Compton scattering enhanced multiple pinhole imager for nuclear medicine." IEEE Trans. Nucl. Sci., vol. 50, pp. 16091617, 2003.

[9] J. A. Fessler, "Mean and variance of implicitly defined biased estimators (such as penalized maximum likelihood): Applications to tomography." IEEE Trans. Image Proc., vol. 5(3), pp. 493-506, 1996.

[10] J. W. Stayman and J. A. Fessler, "Compensation for nonuniform resolution using penalized-likelihood reconstruction in space-variant imaging systems." IEEE Trans. Med. Imag., vol. 23(3), pp. 269-284, 2004.

[11] J. A. Fessler and S. D. Booth, "Conjugate-gradient preconditioning methods for shift-variant image reconstruction." IEEE Trans. Image Proc., vol.8(5), pp. 688-699, 1999.

[12] L. S. Shepp and Y. Vardi, "Maximum likelihood reconstruction for emission tomography." IEEE Trans. Med. Imag., vol. MI-1, pp. 113122,1982

[13] M. H. Hudson and R. S. Larkin, "Accelerated image reconstruction using ordered subsets of projection data." IEEE Trans. Med. Imag., vol. 13(4), pp. 601-609, 1994.

[14] D. Bequé, C. Vanhove, A. Andreyev, J. Nuyts, and M. Defrise, "Correction for imperfect camera motion and resolution recovery in pinhole SPECT." IEEE Med. Imag. Conf. 2004, Rome, Italy, 2004.

[15] J. A. Fessler, "Spatial resolution and noise tradeoffs in pinhole imaging system design: a density estimation approach." Optics Express, vol. 2(6), pp. 237-253, 1998. 